\title{
ABORDAGEM ERGONÔMICA DA MOVIMENTAÇÃO HUMANA, SUA NATUREZA COMPLEXA E ASPECTOS PARA SUA SIMULAÇÃO
}

\section{ERGONOMIC APPROACH OF HUMAN MOVEMENT, ITS COMPLEXITY NATURE AND ASPECTS FOR ITS SIMULATION}

\author{
Henrique Costa Braga ${ }^{1}$, M.Sc. \\ Gray Farias Moita ${ }^{2}, \mathrm{PhD}$. \\ Paulo Eduardo Maciel de Almeida ${ }^{3}$, Dr. \\ (1) Programa de Pós-graduação em Modelagem Matemática e Computacional, CEFET-MG \\ e-mail: bragaseg@yahoo.com.br \\ (2) Programa de Pós-graduação em Modelagem Matemática e Computacional, CEFET-MG \\ e-mail: gray@dppg.cefetmg.br \\ (3) Programa de Pós-graduação em Modelagem Matemática e Computacional, CEFET-MG \\ e-mail: pema@1si.cefetmg.br
}

\begin{abstract}
Movimentação Humana, Ergonomia, Complexidade
No artigo, a movimentação humana, por si só, é apresentada como sendo parte de um sistema ergonômico rico em complexidade. São também incluídos diversos aspectos relevantes envolvidos na movimentação humana, necessários para o desenvolvimento de um modelo computacional. É proposto que esta seja uma modelagem baseada em agentes ergonomicamente situada. Assim, os comportamentos individuais, coletivos e emergentes apresentados na movimentação humana são uma consequência da configuração inicial do sistema, das suas vizinhanças, da autoorganização dos agentes envolvidos e de suas interações locais.
\end{abstract}

\section{Human movement, Ergonomics, Complexity}

In the paper, the human movement is presented as part of an ergonomic system rich in complexity. It is also included several aspects involved in human movement, necessary for the development of a computational model. It is proposed that this is an ergonomically situated agent-based modeling. Thus individual, collective and emergent behaviors shown in human movement are a consequence of the initial system configuration, its neighborhoods, the self-organization of the agents and their local.

\section{Introdução}

Todos nós, cotidianamente, ocupamos e nos movemos em e entre ambientes construídos, seja pelo trabalho, laser, escola, moradia. Na verdade, praticamente passamos a nossa vida inclusa nestes ambientes artificiais. Assim, a nossa interação com estes ambientes influencia sobremaneira a nossa segurança e qualidade de vida. Dentro os diversos aspectos envolvidos na interação com estes ambientes, os relacionados com a movimentação possuem um papel de destaque, já que nos movemos e trocamos continuamente de ambientes, mesmo estando numa mesma edificação ou sistema. Dessa forma, os ambientes devem ser organizados e ter um leiaute apropriado, tanto para se facilitar as movimentações que irão ocorrer, quanto para se minimizar as possibilidades de acidentes. Devido à importância, existe uma série de critérios normativos e legais mínimos a serem considerados na organização e no projeto de edificações, espaços urbanos e outros ambientes a serem ocupados.

Quando a situação em que ocorre a ocupação ou movimentação envolve altas densidades populacionais, a situação pode ficar especialmente perigosa, e cuidados extras devem ser tomados. Estas situações de elevado fluxo ou densidade 


\section{Ergodesign \& HCI}

populacional são cotidianas e passamos periodicamente por elas em momentos e locais tais como auditórios, estádios, igrejas, estações de metro, cinemas, casas de shows. Um caso recente de um grande sinistro em ambiente com alta densidade populacional foi a tragédia ocorrida em 2013 na Boate Kiss, na cidade de Santa Maria - RS, onde 242 pessoas perderam a vida (SILVA FILHO et al., 2013).

Entretanto, mesmo ambientes que não possuem normalmente elevadas densidades populacionais podem se tornar perigosos em situações de escape ou pânico, devido à possibilidade de concentração de pessoas buscando poucas rotas de fuga e pela ocorrência da passagem em pontos não livres (portas, aberturas, escadas, quinas, barreiras, proximidades com paredes, curvas) que podem provocar demoras e colisões na movimentação.

Assim, a existência de programas computacionais que possam auxiliar na verificação das condições de fluidez e segurança de um ambiente torna-se de relevante valor, tanto pela possibilidade de se verificar as mais diversas situações, como também de propor alternativas em termos de leiaute $\mathrm{e}$ organização do ambiente. Estes programas são relevantes como uma ferramenta complementar à aplicação das legislações prescritivas que envolvem o projeto e a concepção de ambientes construídos. Existem já estabelecidos diversos programas para realizar tais simulações (PELECHANO et al., 2008), cada um se utilizando de determinados paradigmas, de forma a serem, na concepção dos seus criadores, o mais apropriado para determinada aplicação e precisão. Um importante paradigma que ainda não foi suficientemente abordado nestes programas é o desenvolvimento de modelos amparados formalmente por uma concepção ergonômica. Espera-se que uma abordagem ergonômica pode propiciar modelagens mais realistas, ao mesmo tempo em que factíveis de serem computacionalmente efetivadas.

Trabalhos na área de segurança do trabalho e ergonomia envolvendo fenômenos complexos são geralmente focados em tarefas ditas sofisticadas. Neste trabalho, a simples movimentação humana, apesar de sua trivialidade aparente, será apresentada como sendo por si só rica em apresentação de comportamentos complexos.
Assim, o objetivo do presente trabalho é apresentar o processo de movimentação humana em um ambiente construído como sendo um sistema ergonômico complexo, exemplificar diversas características ergonômicas que podem ser usadas como balizadoras de modelos computacionais para simular o processo de movimentação humana, e propor as diretrizes para o desenvolvimento de uma modelagem baseada em agentes utilizando a lógica Fuzzy como ferramenta de Inteligência Computacional - IC para emulação da tomada de decisão humana.

\section{Fundamentação}

No contexto deste trabalho, considera-se um ambiente como sendo um sistema aberto, delimitado por fronteiras e composto de diversos elementos que se relacionam dinamicamente entre si, onde uma atividade humana é ou pode ser realizada. A movimentação humana, foco deste trabalho, deve ser considerada como sendo o deslocamento integral do corpo humano, realizado por esforço próprio, entre diferentes regiões do ambiente, mas com possibilidade de eventual suporte mecânico tal como pelo uso de elevadores, escadas rolantes ou cadeiras de rodas. A movimentação humana pode ser tanto considerada como sendo uma atividade para que uma tarefa ou objetivo maior seja alcançado, quanto também como uma própria tarefa em si, constituída por diversas atividades ou comportamentos para ser realizada.

Um sistema pode ser classificado como simples ou complicado. Sistemas simples devem ser fáceis de serem visualizados devido à pequena quantidade de componentes e de suas relações, enquanto os sistemas complicados são difíceis de serem visualizados devido a grande quantidade de componentes e consequentemente de suas relações.

Os sistemas também podem ter diversos comportamentos, tais como comportamento trivial, simples, complexo ou caótico. O comportamento de um sistema não tem necessariamente relação como o tipo de sistema, ou seja, pode-se ter um sistema simples com comportamento complexo, ou pode-se ter um sistema complicado sem apresentar complexidade aparente no seu comportamento. Além disso, o comportamento pode depender tanto de fatores externos ao ambiente ou sistema quanto do estado inicial deste ambiente. Assim, um 


\section{Ergodesign \& HCI}

número especial, volume 5 , ano 5 (2017) ISNN 2317-8876, Rio de Janeiro - Brasil
PUC-Rio Pontifícia Universidade Católica do Rio de Janeiro Departamento de Artes \& Design | PPGDesign

LEUI | Laboratório de Ergodesign e Usabilidade de Interfaces determinado sistema, simples ou complicado, pode eventualmente ter qualquer comportamento (trivial, simples, complexo ou caótico) dependendo da sua estrutura, da vizinhança ou do seu estado inicial.

O estudo e a modelagem de sistemas com comportamentos complexos tem alavancado grande interesse de pesquisadores provenientes das mais diversas áreas tais como física, matemática, engenharia, computação, medicina, sociologia, marketing (BOCCARA, 2004). Tanto na segurança do trabalho quanto na ergonomia, o termo complexidade já tem sido muito empregado e discutido (VIDAL et al., 2002; GRANT, 2002; VIDAL et al., 2009; DANIELLOU et al., 2010; GONZÁLES; SAURIN, 2013). Entretanto, devido à natureza ainda relativamente recente deste modus de aplicação do termo complexidade, tem-se a existência de uma miríade de conceituações para complexidade, tornando-se necessário uma prévia definição deste termo antes de se alavancar o assunto.

No enfoque aqui presente, considerar-se-á estritamente um sistema complexo (ou com comportamento complexo) como aquele que possui elementos interativos e que exibe propriedades emergentes que não resultam da existência de um controlador central (adaptado de BOCCARA, 2004). Neste contexto, propriedades emergentes são características que eclodem como resultado da interação dos elementos que constituem o sistema. Como exemplos de sistemas que podem possuir comportamentos complexos estão algumas implementações de autômatos celulares - AC, como as de Von Neumann, Conway e Wolfram (RUCKER, 2005; CASTRO; CASTRO, 2008). Um AC pode ser definido como um sistema dinâmico discreto que possui as seguintes características fundamentais (BOCCARA, 2004):

- são constituídos por um conjunto discreto de sítios (ou células);

- evoluem em passos discretos de tempo;

- cada sítio assume valores dentro de um conjunto finito de possibilidades;

- os atributos de cada sítio evoluem conforme as mesmas regras; e
- as regras de transição dos atributos de um sítio são função dos atributos de uma vizinhança de sítios.

Na Figura 1 se representa, graficamente, a evolução da implementação de um dos AC unidimensionais de Wolfram (1984). Este é um exemplo de um sistema simples, que, dependendo dos valores de sua entrada, apresenta comportamento complexo.

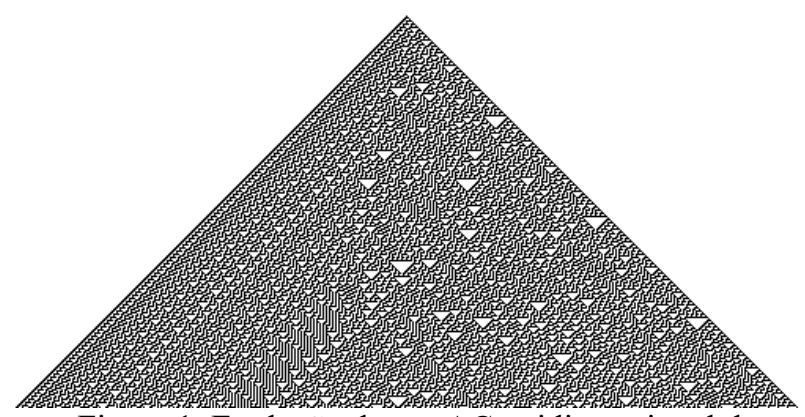

Figura 1. Evolução de um AC unidimensional de Wolfram (1984) como exemplo de um sistema simples apresentando comportamento complexo.

Especificamente na movimentação humana, são muitos os exemplos de comportamentos complexos e dos seus efeitos (STILL, 2000; GOLDSTONE; ROBERTS, 2006).

Na Figura 2, é mostrada uma imagem da entrada de uma escola primária da rede pública localizada em uma via urbana com alto fluxo de veículos. De frente a entrada desta escola, sobre o passeio e separando este passeio da via pública, foi edificada uma parede de forma que quem sai da escola não possa passar reto sobre o passeio e ir direto para a rua, agindo como uma espécie de barreira. Os responsáveis pela escola devem temer que, apesar de certamente darem todas as instruções e advertências para os alunos, pelas características psicofisiológicas desses alunos, pela geometria do local (o passeio possui uma declividade) e talvez também motivados por fatos já ocorridos, no horário de término das aulas e abertura dos portões, alguns alunos simplesmente saiam literalmente correndo da escola diretamente para o meio da rua, portanto correndo alto risco de acidentes. 


\section{Ergodesign \& HCI}

número especial, volume 5 , ano 5 (2017)

ISNN 2317-8876, Rio de Janeiro - Brasil

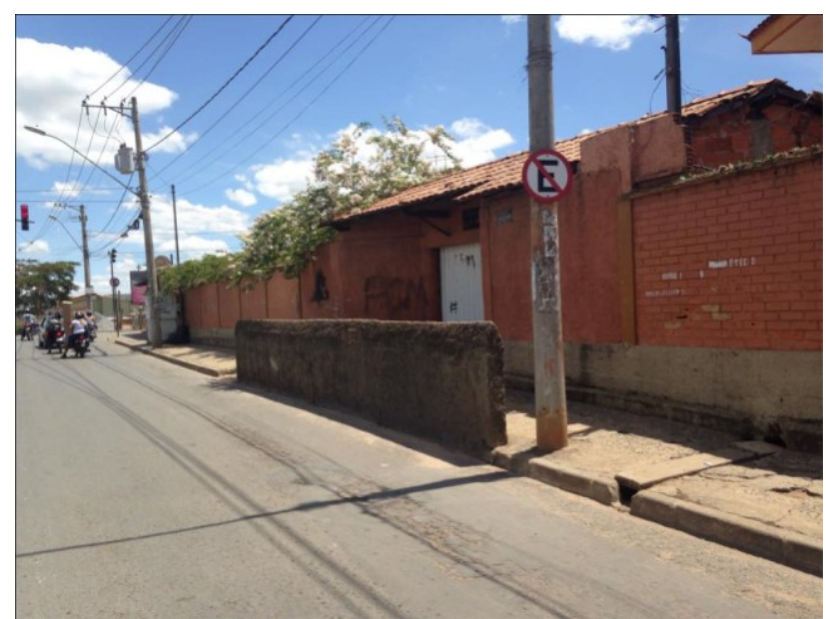

Figura 2. A entrada de uma escola pública fundamental onde uma parede em frente à porta de saída separa $\mathrm{o}$ passeio da via.

Fonte: dos autores.

Também, como exemplo de complexidade na movimentação humana, tem-se a utilização de rotas de deslocamento que não eram previstas ou até mesmo proibidas. Não é raro encontrar em algum local, apesar da existência de uma rota pavimentada apropriada para a movimentação humana, a formação de trilhas que podem ser percebidas pela não existência da vegetação que deveria lá ocorrer (GOLDSTONE; ROBERTS, 2006). Estas trilhas são formadas pela passagem constante de pessoas por este caminho não idealizado para funcionar como rota de passagem humana. Estas rotas alternativas surgem por motivos diversos, sendo os mais comuns à busca pela diminuição da distância a ser percorrida e, para ambientes de maior densidade populacional, da manutenção da velocidade normal de deslocamento.

Outro motivo para a ocorrência das trilhas está na inadequação ergonômica da rota oficial de deslocamento. Na Figura 3 tem-se um exemplo de formação de uma trilha ao lado do caminho dito oficial, onde não há economia no comprimento do percurso. Esta trilha surgiu principalmente pela inadequação do projeto da passagem oficial, formada por blocos de cimento distantes um do outro e em desnível em relação ao piso, de tal modo que torna o andar pelos blocos um processo cansativo.
PUC-Rio Pontifícia Universidade Católica do Rio de Janeiro Departamento de Artes \& Design | PPGDesign

LEUI | Laboratório de Ergodesign e Usabilidade de Interfaces

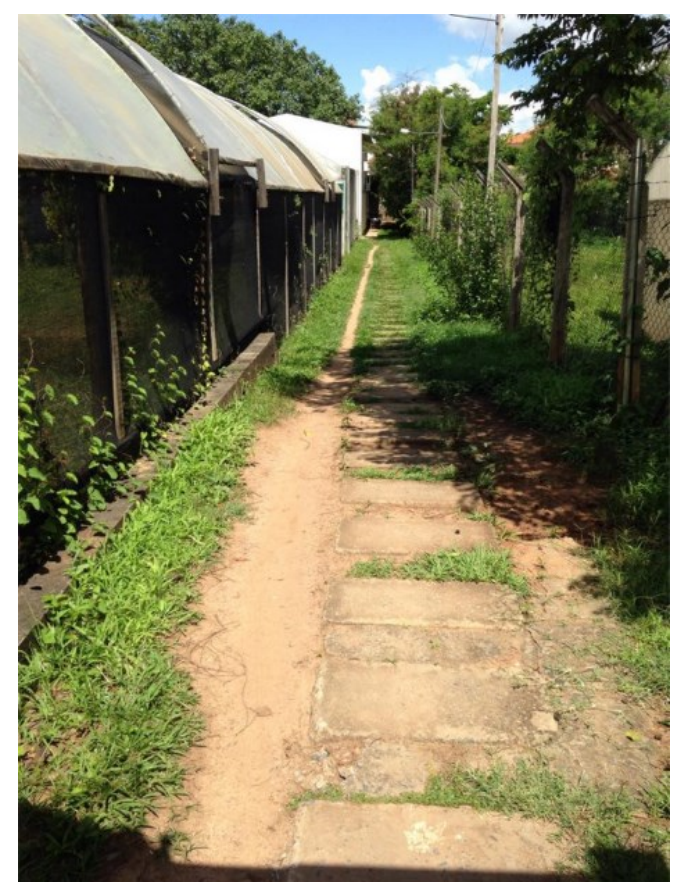

Figura 3. Imagem de uma trilha formada ao lado da passagem oficial.

Fonte: dos autores.

Em situações de emergência, como durante a evolução de um incêndio, a movimentação humana também é rica em efeitos emergentes. Como exemplo se tem o fato de que muitas pessoas, que imediatamente após abandonarem um ambiente em situação de incêndio, reentram no mesmo ambiente, com o incêndio ainda em curso, inclusive pelos motivos mais triviais possíveis (BRYAN; MILKE, 1981), passando a incorrer em sério risco de morte. Outra situação complexa importante que deve ser destacada é a ocorrência do pânico. As pessoas simplesmente não ficam irracionais em situações de grande estresse ou risco iminente. Ações, por mais estranhas que possam parecer, tem seus motivos. Considerar a existência de um comportamento simplesmente irracional para se "justificar" ações que resultam em morte ou graves acidentes tem similaridade com a já discutida e nefasta culpabilidade da vítima em acidentes de trabalho (VILELA et al., 2004).

Assim, incorporar a possibilidade da ocorrência de complexidade na movimentação humana é fundamental para sua análise. Além disso, os parâmetros envolvidos na movimentação humana são diversos e seu estudo deve ser multidisciplinar considerando os mais variados aspectos tais como físicos, ambientais, biológicos, organizacionais e 


\section{Ergodesign \& HCI}

número especial, volume 5 , ano 5 (2017) ISNN 2317-8876, Rio de Janeiro - Brasil
PUC-Rio Pontifícia Universidade Católica do Rio de Janeiro Departamento de Artes \& Design | PPGDesign

LEUI | Laboratório de Ergodesign e Usabilidade de Interfaces mentais. Também possuem sinergia entre si, são individuais e específicos (cada pessoa, situação e sistema são únicos), do grupo (efeitos coletivos e sociais), e dinâmicos (podem mudar radicalmente durante o decorrer do fenômeno).

Comparando as áreas e segmentações da ergonomia (IIDA, 2010) com as facetas envolvidas na movimentação humana percebe-se que elas se confundem. Na movimentação humana, estão envolvidos todos os domínios tradicionais da ergonomia tais como as ergonomias física, cognitiva e organizacional, assim como outros como a ergonomia ambiental. Assim, a movimentação humana está imbuída em um sistema ergonômico verdadeiramente complicado, com possibilidade de possuir comportamento complexo.

A profundidade da abordagem necessária para a completa determinação dos comportamentos complexos observados em uma movimentação humana é de enorme magnitude. Utilizando-se do princípio de Incompatibilidade de Zadeh (ZADEH, 1973):

"conforme a sofisticação de um sistema aumenta, nossa habilidade de fazer afirmações precisas e significantes sobre seu comportamento diminui, até um limiar em que a precisão e a relevância tornam-se praticamente características mutuamente exclusivas" (tradução livre),

pode-se afirmar que a total compreensão determinística de uma movimentação humana é simplesmente impossível de ser realizada.

Entretanto, existe uma alternativa natural: o desenvolvimento de modelos. Um modelo tem a intenção de simplificar os aspectos e relações existentes, mas de forma que ainda tenha relevância para determinado objetivo específico. Ademais, o estudo e modelagem dos sistemas complexos possuem algumas peculiaridades fundamentais que devem ser observadas para uma eventual modelagem, pois, como expressado por Vidal et al. (2002), "alguns aspectos funcionais de sistemas complexos não cabem em um esquema analítico funcionalista". Para se contornar estes pontos, sugere-se a utilização de modelos baseados em agentes.
Quando se fala em modelagem por agentes, o primeiro tipo de modelo que vem a tona são o emprego de AC. A utilização de AC tem permitido com sucesso a realização de modelagens de uma grande gama de sistemas de forma relativamente fáceis em termos de implementação computacional e com possibilidade de se verificar o surgimento de comportamentos emergentes. A própria movimentação de pessoas em ambientes construídos também já foi alvo de modelagens com AC (KIRCHNER; SCHADSCHNEIDER, 2002; BOULMAKOUL; MANDAR, 2011). Contudo, os AC são muito simples e restritos, e uma modelagem estritamente com estes, mesmo que valorosa em relação a diversos aspectos trará limitações para a simulação da movimentação humana.

Para resolver esta situação, sugere-se empregar como paradigma balizador para o desenvolvimento da modelagem baseada em agentes uma abordagem situada da realidade através do emprego de premissas ergonômicas.

Finalmente, de todas as múltiplas fases envolvidas na movimentação humana, uma etapa crítica é a realização da tomada de decisão. Baseado no conjunto de todos os parâmetros, qual a atitude será tomada pela pessoa que está se movendo no ambiente? Esta é uma questão chave para ser respondida. Espera-se que a utilização uma ferramenta de Inteligência Computacional, a Lógica Fuzzy (SIMÕES; SHAW, 2007), possa auxiliar na emulação da tomada de decisão humana necessária para o desenvolvimento do modelo computacional.

\section{Desenvolvimento}

A seguir serão apresentados diversos parâmetros ou fatores, inclusive ergonômicos físicos, cognitivos e organizacionais, de forma a subsidiar uma modelagem da movimentação humana por agentes.

\subsection{Representação do ambiente físico}

Um passo fundamental na modelagem é a da definição de como será realizada a representação do ambiente físico. $\mathrm{O}$ ambiente físico real é tridimensional e contínuo. Entretanto, para simplificar e facilitar a modelagem sugere-se trabalhar com uma vista de topo (os ambientes seriam vistos de forma bidimensional) e discreta (matricial). No caso de ambientes de vários 


\section{Ergodesign \& HCI}

número especial, volume 5 , ano 5 (2017) ISNN 2317-8876, Rio de Janeiro - Brasil
PUC-Rio Pontifícia Universidade Católica do Rio de Janeiro Departamento de Artes \& Design | PPGDesign

LEUI | Laboratório de Ergodesign e Usabilidade de Interfaces patamares, cada patamar pode ser visto de forma independente, bidimensional e discreta, que são unidos aos eventuais demais patamares por caminhos também assim representados.

Uma forma simples de representar esta matriz ambiente é que ela seja retangular e regular (todos os elementos de sua malha representam um espaço igual no mundo real), e cujo valor de cada elemento desta matriz ambiente tenha um significado específico, indicando o que aquele espaço do mundo real representa. Em relação à resolução desta matriz ambiente, quanto menor a resolução maior será a precisão, mas mais elevado também será o custo computacional.

\subsection{Representação das pessoas}

Pessoas são como objetos tridimensionais e uma completa descrição de sua altamente desenvolvida e complicada sequência de movimentos numa marcha humana (VIEL, 2001) não é um assunto trivial. Entretanto, a grande maioria dos pesquisadores desenvolve seus modelos e estudos baseados em duas dimensões, considerando simplesmente a projeção vertical do corpo (PELECHANO et al., 2008), sem levar em consideração o efeito do movimento sobre a forma do perfil do corpo.

Alguns autores consideram que o comprimento dos ombros e a largura do corpo são as medidas primárias requeridas para estudos sobre espaços e instalações para pedestres. Segundo Fruin (adaptado de STILL, 2000): “A profundidade do corpo e largura do ombro são as medidas primárias humanas a serem utilizadas na determinação de espaços para pedestres e instalações. A largura do ombro é o principal fator no projeto de portas e passagens. Muitas escadas são projetadas para permitir a passagem de duas ou mais pessoas, mas na verdade têm largura insuficiente para esta finalidade".

Conforme Cucci Neto (1996), pesquisas feitas nos Estados Unidos consideram que o corpo visto por cima pode se resumir a uma elipse de $60,9 \mathrm{~cm}$ (comprimento do corpo) por 45,7 cm (largura do corpo) tendo uma área média de $0,28 \mathrm{~m}^{2}$ (este valor foi tendenciosamente superior à média real de forma a se compensar, um pouco, as grandes variações dimensionais humanas). Outros autores consideram em suas pesquisas valores inferiores mais realistas, como Fruin (STILL, 2000) que aproxima o corpo humano a uma elipse de $58 \mathrm{~cm}$ por $33 \mathrm{~cm}$, ou Schadschneider et al. (2009) que considera a área média ocupada de $0,2 \mathrm{~m}^{2}$.

Helbing et al. (2000) e Pereira (2007) consideraram em seus estudos o corpo visto por cima como uma simples circunferência ou um quadrado, respectivamente. Still (2000) considerou no seu trabalho duas possibilidades de representação, uma mais realística, e outra mais simples (um quadrado). A representação mais realista foi usada para a representação gráfica, mas para a realização de alguns cálculos mais sofisticados o formato considerado foi simplesmente o quadrado, então considerado como sendo uma espécie de área hipotética de influência. A National Institute of Standards and Technology - NIST (KULIGOWSKI; PEACOCK, 2005) considera em seus modelos uma geometria distinta constituída por três círculos formando o torso e os ombros.

Além disso, existem pessoas que, por características especiais, irão requerer mais espaço para seu deslocamento e passagem, tais como pessoas que se utilizam de muletas, cadeiras de rodas, que transportam crianças em veículos pedestres ou se locomovem com ajuda de um cão guia (PANERO; ZELNIK, 1998; ABNT, 2004).

\subsection{Rotas preferenciais - Wayfinding}

Quando uma pessoa idealiza ou antecipa mentalmente um percurso, ela o faz baseado em vários fatores. Geralmente, o principal fator, e que deverá ser parametrizado, é o de se realizar o percurso na menor distância aparente ou perceptível possível (ou na maior velocidade para atingir o objetivo com o mesmo ou o menor esforço físico). Um algoritmo do tipo "busca em profundidade" pode ser implementado para a determinação de algumas destas rotas preferenciais (PELECHANO et al., 2008).

\subsection{Velocidade de deslocamento}

A determinação da velocidade normal de encaminhamento varia de forma extensiva em relação à população, e mesmo um único indivíduo pode apresentar diferentes velocidades dependendo da situação momentânea. Abley (2007) destaca os seguintes fatores: idade, condição física e sexo do indivíduo; familiaridade da rota; finalidade do 
Ergodesign \& HCI

número especial, volume 5 , ano 5 (2017) ISNN 2317-8876, Rio de Janeiro - Brasil
PUC-Rio Pontifícia Universidade Católica do Rio de Janeiro Departamento de Artes \& Design | PPGDesign

LEUI | Laboratório de Ergodesign e Usabilidade de Interfaces encaminhamento; comprimento do percurso; características da rota (largura de passagem, tipo de pavimentação, mudança de nível); condições de visibilidade; condições meteorológicas; se sozinho ou acompanhado; densidade populacional; sentido do fluxo; barreiras diversas; e aspectos cognitivos. Acrescenta-se também a estatura como um fator importante (OKUNO et al., 1986, p. 454). Fatores angustiantes podem induzir uma aceleração da velocidade de deslocamento, similar ao que pode ocorrer em algumas situações de trabalho (ROSSI et al., 2009).

Uma possibilidade para a determinação da velocidade média de deslocamento está na sua relação com a percepção que a pessoa tem da distância entre ela mesma e algum obstáculo à frente (THOMPSON; MARCHANT, 1995). Na Figura 4 se tem a relação entre a velocidade "normal" de deslocamento em função da distância a outra pessoa. Observa-se que a partir de um limiar, a velocidade de encaminhamento se eleva de forma linear com o aumento da distância entre pessoas, até que em certo ponto (cerca de $90 \mathrm{~cm}$ ) começa a diminuir o ritmo de seu crescimento até se estabilizar em um valor fixo para distâncias entre pessoas acima de aproximadamente 1,6 $\mathrm{m}$. Os valores da velocidade de deslocamento variam em patamares diferentes dependendo da população específica em estudo, mas seguindo um formato de curva característico.

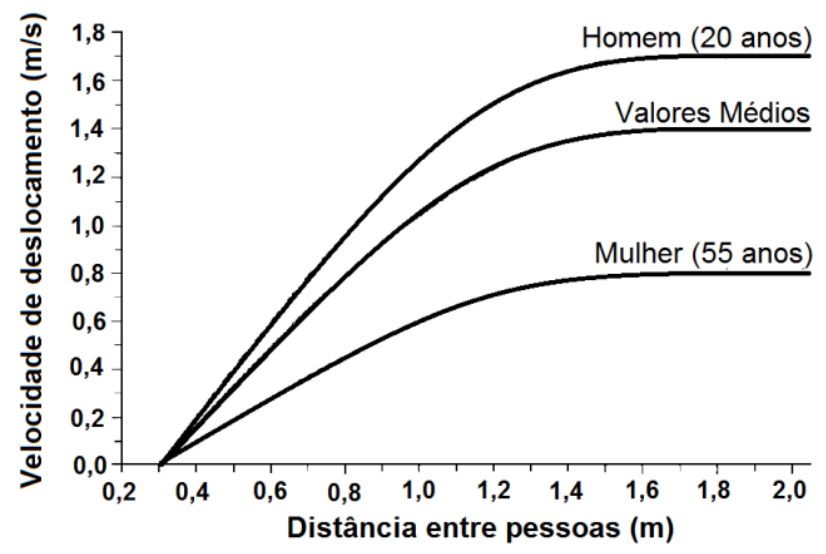

Figura 4. Relação entre a velocidade de deslocamento em função da distância entre pessoas.

Fonte: adaptado de Thompson; Marchant (1995).

\subsection{Nível de estresse}

Obviamente que as questões psicológicas e cognitivas são fundamentais em qualquer abordagem ergonômica mais completa (VIDAL;
CARVALHO, 2008; VACONCELOS et al., 2010). Uma destas questões é saber como o nível de estresse pode influenciar numa tomada de decisão. Staal (2004) considera que a habilidade de uma tomada de decisão varia com o nível de estresse a qual a pessoa está submetida conforme um "U" invertido (Figura 5).

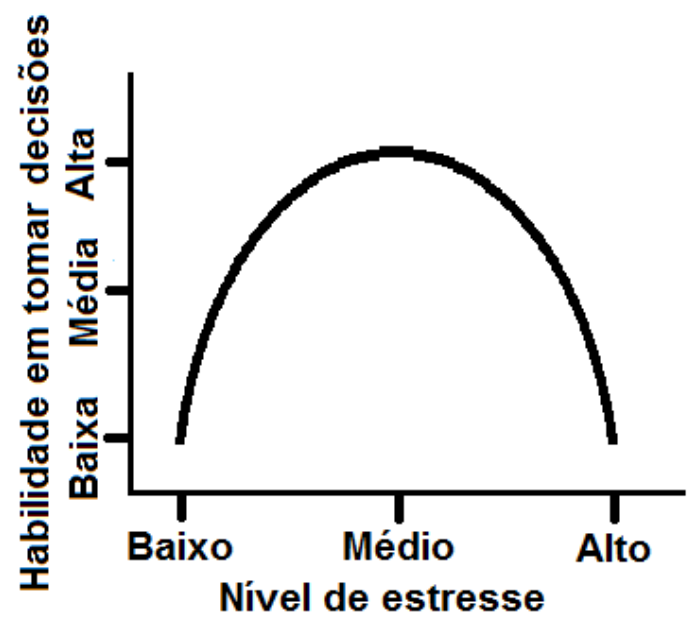

Figura 5. Variação da habilidade em se tomar decisões com o nível de estresse.

Fonte: adaptado de Staal (2004).

Assim, segundo esta hipótese, as pessoas sujeitas a um nível de estresse intermediário tomam melhores decisões do que as pessoas sujeitas a um nível de estresse considerado baixo ou considerado elevado. Adicionalmente, em caso de nível de estresse muito alto, pode acontecer nas pessoas um comportamento denominado de não adaptativo, onde ocorre uma perda ou deterioração dos acordos sociais normalmente praticados (PAN et al., 2007). A resposta ao nível de estresse é uma questão individual (em situações de emergência, um bombeiro treinado poderá ter uma resposta e comportamento diferente do apresentado por uma pessoa não treinada) e influenciada pela situação.

\subsection{Zona de conforto}

Em situações de médio estresse, procura-se manter a velocidade normal de deslocamento. Nesta situação, normalmente se procura evitar andar demasiadamente perto demais de paredes e obstáculos. Isto está relacionado a fatores como busca de segurança, já que um choque eventual com um obstáculo pode ser evitado se a movimentação ocorrer a certa distância do obstáculo (STILL, 


\section{Ergodesign \& HCl}

número especial, volume 5 , ano 5 (2017) ISNN 2317-8876, Rio de Janeiro - Brasil
PUC-Rio Pontifícia Universidade Católica do Rio de Janeiro Departamento de Artes \& Design | PPGDesign

LEUI | Laboratório de Ergodesign e Usabilidade de Interfaces
2000), ou sociais, como a busca por um espaço de conforto (PAN et al., 2007).

Outra situação relacionada com a existência de zonas de conforto são no caso da movimentação acontecer como algum grupo social coeso, como em família, onde se pode procurar manter a ocorrência de uma movimentação em conjunto. Isto pode ser mais complicado em situações de médio e alto estresse.

\subsection{Efeito inércia}

Uma vez iniciado um percurso como resultado de uma decisão tomada, tendo sido alterados os estímulos que resultaram neste movimento, um novo movimento pode não acontecer imediatamente, podendo haver uma tendência de se manter o movimento anterior, pelo menos durante algum tempo.

Além disso, numa movimentação as pessoas reagem continuamente ao observado no ambiente (assim como o restante do ambiente também é influenciado continuamente pelo comportamento das pessoas, tudo ao mesmo tempo). Entretanto, dependendo dos estímulos recebidos ou gerados, uma mesma ação pode não ser alterada a todo tempo. Uma pessoa toma uma decisão de certo caminho a seguir (pode ser poucos passos), e durante este trajeto, não recebendo novos estímulos, simplesmente o mantem e muda seu foco principal para outras decisões. Recebendo um novo estímulo (ou cessando o percurso definido), volta a ter como foco um novo trajeto a seguir.

\subsection{Tempo de reação em emergência}

Quando uma situação de emergência se inicia, as pessoas irão levar um tempo para perceberem o acontecimento ( $\boldsymbol{t}_{r}-$ tempo de reconhecimento), outro tempo para iniciarem efetivamente uma reação ( $\boldsymbol{t}_{a^{-}}$- tempo de resposta ou ação) e ainda o tempo efetivo de escape ( $\boldsymbol{t}_{\boldsymbol{m}}$ - tempo de movimento). Este somatório de tempos ( $\boldsymbol{t}_{T^{-}}$tempo total gasto) deve ser considerado na concepção de um ambiente (GOUVEIA; ETRUSCO, 2002), onde:

$$
t_{T}=t_{r}+t_{a}+t_{m}
$$

Este tempo de reação é muito variável de pessoa para pessoa (por força da idade, concentração, características físicas e cognitivas), além de depender de como a situação de emergência é percebida pelos envolvidos (sinal sonoro, movimentação de outras pessoas, cheiro ou sinal de fumaça, visualização de algum perigo e da sua distância ao mesmo, percepção do risco). O tempo de resposta pode variar de alguns segundos a até mesmo horas (GOUVEIA; ETRUSCO, 2002).

\subsection{Sinalização visual e sonora}

A sinalização visual é um aspecto importante em um ambiente. Ela serve como indicativo para direcionar aqueles que não sabem localizar os pontos de interesse, como, por exemplo, as saídas de emergência ou pontos de refúgio, tanto em situações de normalidade quanto em caso de anormalidade, como falta de iluminação ou bloqueio por fumaça. Também é muito importante para indicar a localização dos equipamentos de combate a incêndio, tais como hidrantes e extintores, ou como avisos de alerta ou instruções de segurança e conduta. Assim, a interação das pessoas com a sinalização pode ter um papel muito importante no processo de movimentação.

Entretanto, com relação à sinalização visual, destacam-se alguns problemas. Em certos ambientes, como alguns grandes centros comerciais, apesar da existência da sinalização de emergência, a sua devida visualização pode ser dificultada pela eventual enorme quantidade de outros tipos de sinalizações (ou estimulações visuais) existentes (excesso de poluição visual). Isto dificulta tanto a própria localização da sinalização de emergência, como também sua devida interpretação. Outro ponto é que, apesar de existir disponível toda uma tecnologia apropriada capaz de auxiliar para que a sinalização possa ser eficaz, mesmo em algumas situações críticas, em muitos casos a sinalização efetivamente aplicada é de baixa qualidade servindo apenas para atendimento mínimo quanto às exigências dos requerimentos prescritivos legais. A manutenção apropriada da sinalização é outro fator importante, cujos responsáveis pelo ambiente não devem se descuidar.

A sinalização sonora também possui um importante papel, mas o público alvo da sinalização sonora 


\section{Ergodesign \& HCI}

número especial, volume 5 , ano 5 (2017) ISNN 2317-8876, Rio de Janeiro - Brasil
PUC-Rio Pontifícia Universidade Católica do Rio de Janeiro Departamento de Artes \& Design | PPGDesign

LEUI | Laboratório de Ergodesign e Usabilidade de Interfaces deve estar devidamente condicionado para reconhecê-la e agir conforme o esperado.

\subsection{Quedas e acidentes}

Quedas e acidentes na movimentação não podem ser desconsiderados; pelo contrário, tem um papel importante. As consequências podem ser desde um desequilíbrio momentâneo sem queda (efeito malabarista), a até mesmo uma grave lesão ou morte. Acidentes podem acontecer com pessoas de qualquer idade, mas quanto mais idosa a pessoa maior o risco.

Na Figura 6, elaborada baseada em dados apresentados por Abley (2007), se apresenta a quantidade relativa de internações hospitalares relativas a quedas em função da idade. Nota-se o grande aumento da possibilidade de quedas com o avanço da idade, sendo os idosos os mais suscetíveis a graves acidentes durante a movimentação.

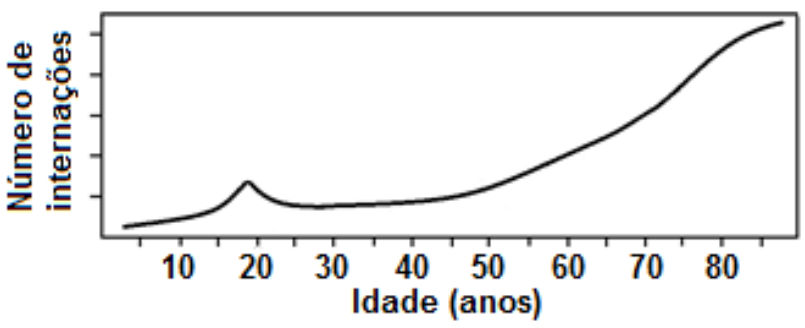

Figura 6. Quantidade relativa de internações hospitalares causadas por quedas em função da idade do acidentado. Fonte: dos autores, baseada em Abley (2007).

Outros grupos de risco importantes são os adolescentes e de pessoas sob o efeito de drogas ou álcool que, em uma situação de pânico ou superpopulação, favorecem o acontecimento de acidentes.

\subsection{Tipo e qualidade do piso}

O tipo e qualidade do piso também podem favorecer a ocorrência de acidentes, por escorregões, tropeções ou desequilíbrios. Evidentemente que uma mudança de nível (escada, passarela, degrau, descontinuidades, defeitos, etc.) impacta nas condições da movimentação. Entretanto, mesmo num mesmo nível, o tipo e a qualidade do piso têm grande relevância. Diferentes tipos de piso (concreto, asfalto, pedras, tacos, madeira, blocos artificiais, agregados, grama, terra, brita, areia), assim como sua condição (molhado, seco, sujo), propiciam diferentes características e dificuldades de movimentação e segurança que devem ser considerados.

A existência de defeitos ou desníveis que podem passar despercebidos são um fator agravante e podem provocar acidentes. Bird et al. (2006) estudou esta relação e verificou que para defeitos não percebidos de $6 \mathrm{~mm}$, cerca de $10 \%$ dos transeuntes podem tropeçar nos mesmos; para defeitos de $15 \mathrm{~mm}$, cerca de $50 \%$ dos transeuntes poderão tropeçar; para defeitos de $40 \mathrm{~mm}$, quase a totalidade dos transeuntes poderão tropeçar; e, já para defeitos maiores de $40 \mathrm{~mm}$, a probabilidade de um transeunte tropeçar diminui devido a maior visibilidade do defeito (Figura 7).

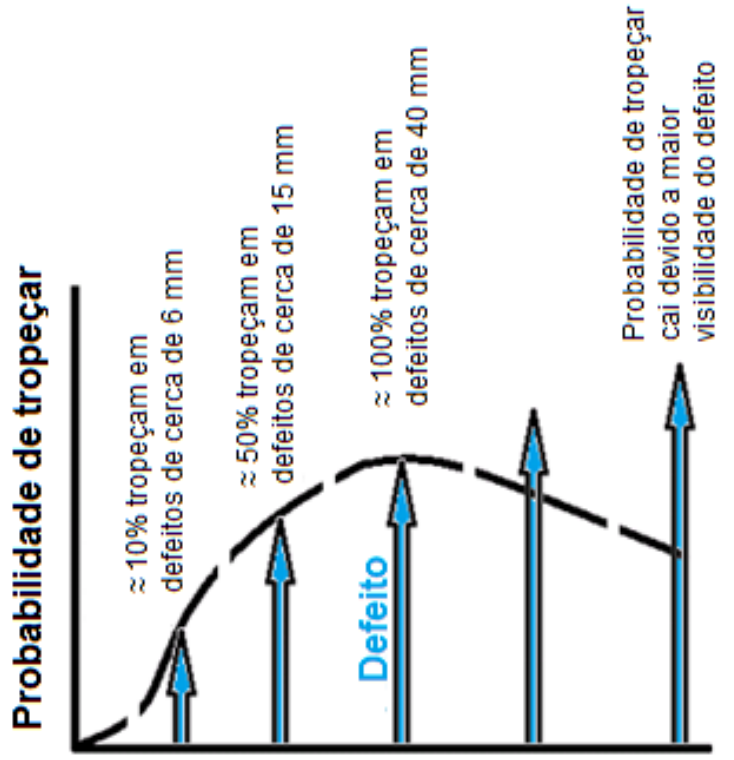

Altura do defeito

Figura 7. A probabilidade da ocorrência de um tropeção em função da altura de um defeito não percebido. Fonte: dos autores, baseada em Bird et al. (2006).

\subsection{Fatores organizacionais}

Estes fatores tem uma importância primordial e não podem ser deixados de lado na concepção de um ambiente. Um ambiente pode ser devidamente projetado, mas a organização pode com o tempo alterar as suas características ou não efetuar as manutenções devidas. Estas alterações podem ocorrer por vários motivos, tais como econômicos, por desconhecimento, ou para minimização dos riscos. Os responsáveis devem ser treinados sobre a importância da manutenção do ambiente conforme 


\section{Ergodesign \& HCI}

número especial, volume 5 , ano 5 (2017) ISNN 2317-8876, Rio de Janeiro - Brasil
PUC-Rio Pontifícia Universidade Católica do Rio de Janeiro Departamento de Artes \& Design | PPGDesign

LEUI | Laboratório de Ergodesign e Usabilidade de Interfaces previamente planejado, e mesmo mínimas alterações, tanto no leiaute como na ocupação, somente podem ocorrer após uma reanálise do projeto da edificação por parte de profissional especializado.

Como exemplos de interferências organizacionais podem ser citados: a não formação ou reciclagem da brigada de emergência; a não inspeção e manutenção, ou uma alteração, nos sistemas de proteção, ou da própria edificação, ou dos seus elementos e sistemas (elétricos, hidráulico, gás, estrutural, etc.); bloqueio de saídas de emergência ou dos equipamentos de proteção; alterações do leiaute ou ocupação; interdição parcial ou total das rotas de fuga; alteração da carga de incêndio, não controle da população, dentre outras.

\subsection{Outros fatores relevantes}

São diversos os outros fatores envolvidos, tais como os fatores ambientais, coletivos e sociais, que podem também influenciar na modelagem pretendida. Fatores ambientais, e assemelhados dos mais diversos, tais como ruído, temperatura, fumaça, umidade, iluminação, vapores, gases, vibração, além de alterarem a segurança do ambiente, também influenciam no comportamento humano. Em relação aos efeitos coletivos, são muitas as possibilidades de ocorrência na movimentação humana.

Como exemplo uma edificação pode possuir duas ou mais saídas de emergência, que em princípio atenderiam as necessidades desta edificação, mas por efeitos coletivos a maioria da população pode buscar durante uma evacuação uma única saída ou rota de fuga, que ficará sobrecarregada, enquanto as outras ficam subutilizadas. Fatores sociais também são importantes. Pessoas se movendo como um grupo social (uma família, por exemplo) tendem a se manter coesas, o que pode ser positivo no que se refere a segurança dos elementos mais frágeis deste grupo, mas que também pode trazer riscos a todo o grupo.

\section{Discussões}

Na Figura 8 se apresenta uma representação esquemática dos diversos parâmetros envolvidos na movimentação humana e suas inter-relações. Diversos destes parâmetros foram anteriormente tratados. Para que se possa realizar a simulação, estes parâmetros precisam ser incorporados em um algoritmo computacional e valorados dentro de um sistema de tomada de decisão. Existe uma enorme gama de possibilidades de como desenvolver o modelo, mas duas características geralmente antagônicas são importantes, a simplicidade e a qualidade, visando à obtenção de um baixo custo computacional na sua implementação associado com a possibilidade da obtenção de bons resultados na simulação.

Na modelagem baseada em agentes, cada pessoa simulada terá incorporadas suas características, e irá interagir com o ambiente, percebendo deste ambiente suas propriedades, e tomando por sua própria decisão a escolha do roteiro e forma de movimentação. Esta forma de modelagem não obrigará nenhuma das pessoas simuladas a tomarem uma decisão; o processo decisório é individual e particular, e deste comportamento individual esperase obter os efeitos coletivos e complexos que ocorrem numa movimentação real via uma autoorganização do sistema e de suas relações locais.

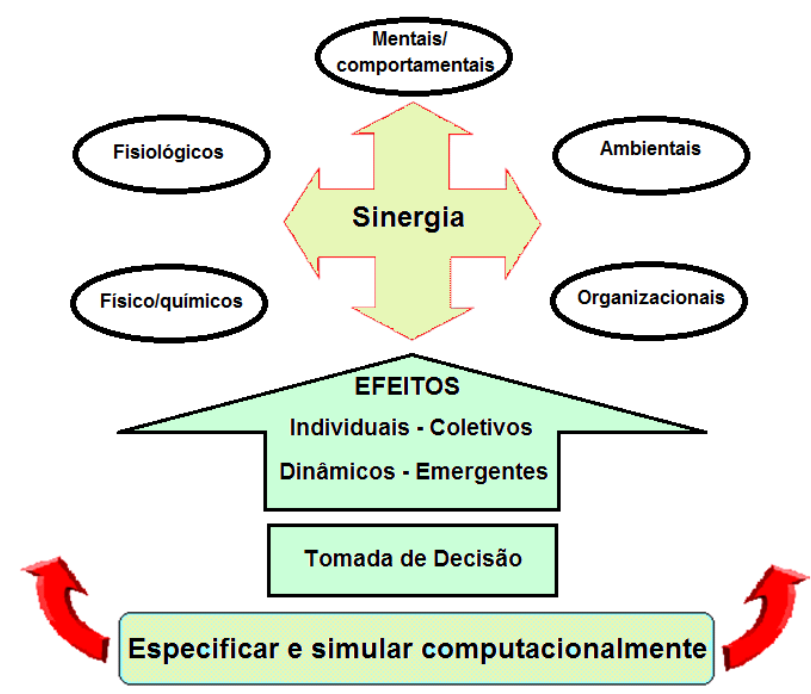

Figura 8. Representação esquemática de diversos parâmetros e suas inter-relações envolvidas na movimentação humana.

Fonte: dos autores.

Na Figura 9 é apresentado um esquema desta relação entre a auto-organização entre os agentes baseada em relações locais e sua influência no sistema como um todo. 


\section{Ergodesign \& HCI}

número especial, volume 5 , ano 5 (2017) ISNN 2317-8876, Rio de Janeiro - Brasil
PUC-Rio Pontifícia Universidade Católica do Rio de Janeiro Departamento de Artes \& Design | PPGDesign

LEUI | Laboratório de Ergodesign e Usabilidade de Interfaces

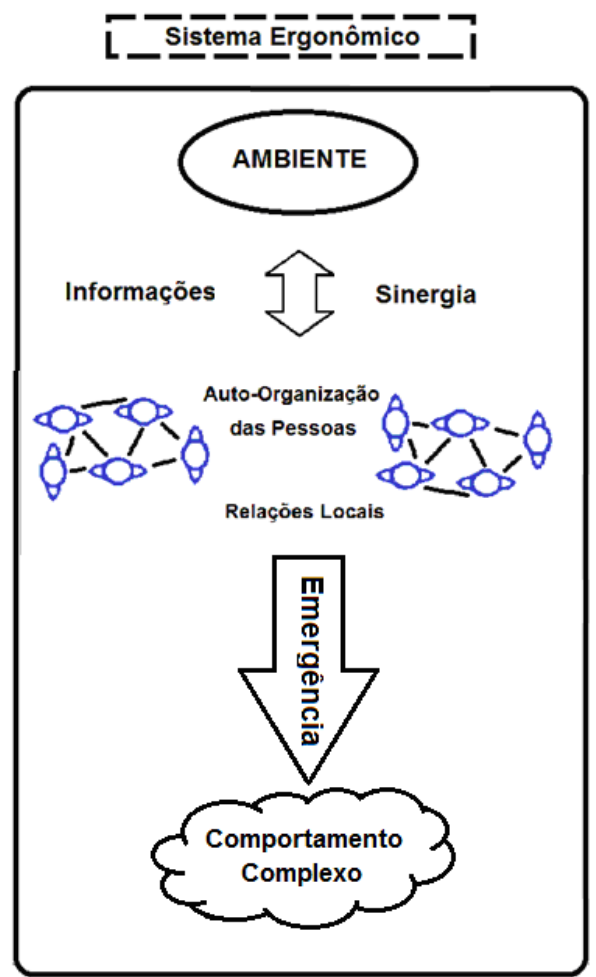

Figura 9. Representação da auto-organização dos agentes por relações locais no sistema.

Fonte: dos autores.

Tal proposição de modelagem ergonômica baseada em agentes já foi implementada computacionalmente com resultados promissores (BRAGA et al., 2014), inclusive a observação de alguns fenômenos complexos, mas de extensão ainda limitada. Nessa modelagem foram incorporadas as grandezas rotas preferenciais (wayfinding), nível de estresse, velocidade normal de deslocamento, efeito inércia e efeito pessoas, dentro de um ambiente discretizado na forma de uma matriz retangular com $9 \mathrm{~cm}$ de resolução, e usando a lógica fuzzy para emulação da tomada de decisão humana. Em trabalhos futuros pretende-se incrementar a profundidade e quantidade de propriedades envolvidas e de suas inter-relações.

\section{Conclusões}

Neste trabalho se demonstrou que a movimentação humana, mesmo por si só, já está inclusa em um sistema ergonômico, onde os aspectos físicos, organizacionais, cognitivos e ambientais se fazem extensamente presentes. Também se demostrou que, apesar da movimentação humana ser aparentemente simples e corriqueira, a complexidade, ou o surgimento de fenômenos emergentes, tem um papel muito importante no seu desenvolvimento. A modelagem baseada em agentes, elaborada de forma ergonomicamente situada, aparenta ser uma poderosa ferramenta computacional para simulação da movimentação humana em um contexto de complexidade, onde relações locais poderão gerar auto-organização.

\section{Referências}

ABLEY, S. Walkability tools research: variables, collection and methodology, Nova Zelândia: LNTZ, abr., 2007, 65 p.

\section{ASSOCIAÇÃO BRASILEIRA DE NORMAS TÉCNICAS. ABNT NBR 9050:2004 -} acessibilidade a edificações, mobiliário, espaços e equipamentos urbanos. Rio de Janeiro, dez., 2005, $97 \mathrm{p}$.

\section{BAPTISTA, A.H.N. Proposição da teoria da} acessibilidade efetiva com plano de verificação para estruturas de circulação de pedestre, Recife: Programa de Pós-graduação em Desenvolvimento Urbano: Universidade Federal de Pernambuco, 2010, $325 \mathrm{p}$. Tese (doutorado).

BIRD. S.; SOWERBY, C.R.; ATKINSON, V.M.

Development of a risk analysis model for footways and cycle tracks, Berkshire: UK: Transport Research Laboratory, 2006, 74 p. Published Project Report TRL 171.

BOCCARA, N. Modeling complex systems, Springer, 2004. 397 p.

BOULMAKOUL, A.; MANDAR, M. Fuzzy Ant Colony Paradigm for Virtual Pedestrian Simulation, The Open Operational Research Journal, n. 5, 2011, p. 19-29.

BRAGA, H.C.; MOITA, G.F.; CAMARGO, F.; ALMEIDA, P.E.M. Simulação da movimentação de pessoas em situações de emergência: aspectos ergonômicos e computacionais com autômatos fuzzy e sua aplicação ao projeto arquitetônico. Ambiente Construído, v. 14, n. 2, 2014, p. 61-77.

BRYAN, J.L.; MILKE, J.A. The determination of behavior response patterns in fire situations, Project People II. Final report - health care. 


\section{Ergodesign \& HCI}

número especial, volume 5 , ano 5 (2017) ISNN 2317-8876, Rio de Janeiro - Brasil
PUC-Rio Pontifícia Universidade Católica do Rio de Janeiro Departamento de Artes \& Design | PPGDesign

LEUI | Laboratório de Ergodesign e Usabilidade de Interfaces
Maryland: University of Maryland: NBS. 1981, 304 p. NBS-GCR-81-343.

CASTRO, M.L.A.; CASTRO, R.O., Autômatos Celulares: implementações de Von Neumann, Conway e Wolfram. Revista de Ciências Exatas e Tecnologia, v. 3, n. 3, 2008. p. 89-106.

CUCCI NETO, J. Aplicações da engenharia de tráfego na segurança dos pedestres, São Paulo: Escola Politécnica: Universidade de São Paulo, 1996, 188 p. Dissertação (Mestrado em Engenharia de Transportes).

DANIELLOU, F.; SIMARD, M.; BOISSIÈRES, I. Facteurshumains et organisationnels de la sécurité industrielle: um état de l'art. Toulouse: FonCSI. 2010, 124 p. Numéro 2010-2 des Cahiers de la Sécurité Industrielle.

GOLDSTONE, R.L.; ROBERTS, M.E. Selforganized trail systems in groups of humans, Complexity, v. 11, n. 6, 2006, p. 43-50.

GONZÁLEZ, S.S.; SAURIN, T.A. Princípios para gestão de procedimentos em sistemas sócio-técnicos complexos, Ação Ergonômica, v. 8, n. 1, 2013, p. 48-62.

GOUVEIA, A.M.C.; ETRUSCO, P. Tempo de escape em edificações: os desafios do modelamento de incêndio no Brasil, REM - Revista da Escola de Minas, v. 55, n. 4, out-dez, 2002, p. 257-261.

GRANT, C.B. Theory of complex communication, Ação Ergonômica, v. 1, n.3, 2002, p. 21-37.

HELBING, D.; FARKAS, I.; VICSEK, T. Simulating Dynamical Features of Escape Panic, Nature, v. 407, set., 2000, p. 487-490.

IIDA, I. Ergonomia: projeto e produção, 2a ed., São Paulo: Edgard Blücher, 2010, 614 p.

KIRCHNER, A.; SCHADSCHNEIDER, A. Simulation of evacuation process using a bionicsinspired cellular automaton model for pedestrian dynamics, Physica A, n. 312, 2002, p. 260-276.

KULIGOWSKI, E.; PEACOCK, R.D. A review of building evacuation models, NIST, 2005, $156 \mathrm{p}$. Tecchnical Note 1471.
NUNES, I.L. Handling human-centered systems uncertainly using Fuzzy Logics - a review, The Ergonomics Open Journal, n. 3, 2010, p. 38-48.

OKUNO, E.; CALDAS, I.L.; CHOW, C. Física para ciências biológicas e biomédicas, São Paulo: Harbra, 1986, $490 \mathrm{p}$.

PAN, X.; HAN, C.S.; DAUBER, K.; LAW, H. A Multi-agent Based Framework for the Simulation of Human and Social Behaviors during Emergency Evacuations, AI and Society, n.2, v. 22, 2007, p. 113-132.

PANERO, J.; ZELNIK, M., Las dimensiones humanas em los espacios interiores, México: G. Gili, 8a ed., 1998, 320 p.

PELECHANO, N., ALLBECK, J.; BADLER, N. Virtual Crowds: methods, simulation, and control. San Rafael: Morgan \& Claypool Publishers, 2008.

PEREIRA, L. Estudo do tempo de evacuação total em ambientes gerais via autômatos finitos. Belo Horizonte: Departamento de Estatística: UFMG, 2007, 63 p. Dissertação (Mestrado em Estatística).

ROSSI, E.Z.; MENDES, A.M.; SIQUEIRA, M.V.; ARAÚJO, J.N.G. Sedução e servidão em um caso de LER/DORT: diálogo entre a Psicodinâmica do Trabalho e a Sociologia Clínica, Psicologia Política, v. 9 , n. 18,2009 , p. 313-330.

RUCKER, R. The lifebox, the seashell and the soul. New York: Thunder's Mouth Press. 2005, 149 p.

SCHADSCHNEIDER, A.; KLINGSCH, W.; KLUPFEL, H.; KRETZ, T.; ROGSCH, C.; SEYFRIED, A. Evacuation dynamics: empirical results, modeling and applications. In: Encyclopedia of Complexity and System Science. Ed. B. Meyers, Springer, Berlim, 2009. 57 p.

SILVA FILHO, L.C.P.; WENGROVER, C.; SALDANHA, M.; BRENTANO, T.; RODRIGUES, E.E.C. Análise do sinistro na Boate Kiss, em Santa Maria, RS. Porto Alegre: CREA-RS. 2013.

SIMÕES, M.G.; SHAW, I.S. Controle e modelagem Fuzzy, $2^{a}$ ed., São Paulo: Blücher: FAPESP, 2007, $186 \mathrm{p}$. 


\section{STAAL, M.A. Stress, cognition and human} performance: a literature review and conceptual framework. NASA, 2004. $171 \mathrm{p}$.

STILL, G.K. Crowd dynamics. Warwick: Department of Mathematics: University of Warwick, 2000, 280 p. Tese (Doctor of Philosophy in Mathematics).

THOMPSON, P.A.; MARCHANT, E.W. Testing and application of the computer model "SIMULEX", Fire Safety Journal, n. 24, 1995, p. 149-166.

VASCONCELOS, C.S.F.; VILLAROUCO, V.; SOARES, M.M. Contribuição da psicologia ambiental na análise ergonômica do ambiente construído. Ação Ergonômica, n. 5, v. 3, 2010, p. 14-20.

VASCONCELOS, R.C.; LIMA, F.P.A.; CAMAROTTO, J.A.; ABREU, A.C.M.S.; COUTINHO FILHO, A.O.S. Aspectos de complexidade do trabalho de coletores de lixo domiciliar: a gestão da variabilidade do trabalho na rua, Gestão \& Produção, v. 15, n. 2, 2008, p. 407419.

VIDAL, M.C.; BONFATTI, R.J.; CARVÃO, J.M. Ação ergonômica em sistemas complexos proposta de um método de interação orientada em situação: a conversa-ação, Ação Ergonômica, v. 1, n. 3, 2002, p. 39-64.
VIDAL, M.C.; CARVALHO P.V.R. Ergonomia cognitiva: raciocínio e decisão no trabalho. Rio de Janeiro: EVC, 2008. 192 p.

VIDAL, M.C.; CARVALHO, P.V.R.; SANTOS, M.S.;SANTOS, I.J.L. Colletive work and resilience of complex sistems, Journal of Loss Prevention in the Process Industries, v. 22, n. 4, 2009, p. 516527.

VIEL, É. A marcha humana, a corrida e o salto: biomecânica, investigações, normas e disfunções. Barueri: Manole, 2001. 277 p.

VILELA, R.A.G.; IGUTI, A.M.; ALMEIDA, I.M. Culpa da vítima: um modelo para perpetuar a impunidade nos acidentes de trabalho. Cadernos de Saúde Pública, v. 20, n. 2, 2004, p. 570-579.

WOLFRAM, S. Cellular automata as models of complexity, Nature, v. 311, 1984. p. 419-424.

ZADEH, L.A. Outline of a new approach to the analysis of complex systems and decision processes, IEE Transactions on Systems, Man and Cybernetics, v. 3, n. 1, 1973, p. 28-44.

\section{Agradecimentos}

Os autores deste trabalho agradecem ao CEFET-MG e a CAPES pelo suporte obtido. 